\title{
La politique du multilinguisme dans le système éducatif polonais
}

\section{Multilingual policy in the Polish education system}

\begin{abstract}
The aim of this article is to reflect on the implementation modalities of educational policy promoting multilingualism in Poland, a country where linguistic and cultural variety remains relatively low. The article begins with a definition of the concept of "language policy". Subsequently, the European Union's directives promoting the policy of multilingualism in the educational context are presented. In this part, the focus is on the following aspects: modern foreign languages, regional and minority languages, certification of language skills and teaching of a curriculum subject integrated into foreign language instruction. The last part of the article concentrates on the implementation of the said directives in Poland. In the research, the author relies on statistical data from various sources to demonstrate that - even if Poland is considered a country where linguistic and cultural variety remains low - foreign (in a broad sense of the term), regional and minority languages are regaining their rightful place in the functioning of the state, including in the Polish education system.
\end{abstract}

KEYWORDs: language policy, multilingualism, school, Poland.

Mots-CLÉs : politique linguistique, multilinguisme, école, Pologne.

\section{INTRODUCTION}

L'objectif du présent article est de présenter la notion de politique linguistique sur l'exemple de la Pologne dont le paysage linguistique peut sembler peu varié. Pour ce faire, nous définirons brièvement le concept de politique linguistique, tout en mettant l'accent sur son caractère dynamique et multidimensionnel. Ensuite, nous présenterons les axes de ladite politique, promus par l'Union européenne, à savoir : l'enseignement/apprentissage des langues étrangères vivantes, régionales et minoritaires, de même que les principes de l'enseignement d'une matière intégré à une langue (EMILE). Cela nous permettra de passer à la présentation de quelle façon les principes ci-mentionnés sont mis en place en Pologne, un des pays membres de l'UE depuis 2004. Comme la Pologne est un 
pays où les diversités linguistique et culturelle sont peu variées, la question de la politique linguistique, analysée du point de vue éducatif, nous paraît intéressante. Dans notre texte, nous nous référerons à des données venant de sources diverses. C'est la raison pour laquelle elles ne sont pas toujours complètes ou peuvent paraître anciennes. Force est quand même de constater qu'il est difficile d'accéder aux données les plus récentes relatives à l'enseignement/apprentissage des langues minoritaires/régionales, car c'est une question souvent négligée par les autorités éducatives. Il en résulte que dans l'article, nous ne nous référons qu'à des données disponibles pour montrer le paysage linguistique de la Pologne dans le contexte éducatif, ce qui constitue le noyau central de nos réflexions. Pour la même raison, nous ne nous concentrons pas non plus sur les approches didactiques qui pourraient être mises en place dans le cadre de la politique du multilinguisme. Nous sommes persuadé qu'elles pourraient constituer un champ $\mathrm{d}^{\prime}$ investigations à part, d'autant plus que les recherches concernant le sujet en question sont peu nombreuses, surtout dans le contexte éducatif polonais.

\section{LA NOTION DE POLITIQUE LINGUISTIQUE}

Aborder la question de la politique linguistique nécessite de se mettre d'accord sur le vocable en question. Comme le remarque - à juste titre - J.-C. Beacco (2016 : 10-12), la notion de politique linguistique puise dans les apports de la sociolinguistique qui « peut être appréhendée globalement en tant qu'une étude du langage comme fait social ou en relation avec la société » (Beacco 2016 : 11). En effet, les résultats de plusieurs recherches menées dans le cadre de la sociolinguistique - dans le sens large du terme - peuvent être exploités lors de l'élaboration des politiques linguistiques. Il est notamment question des domaines de recherches tels que : les contacts des langues, le phénomène de véhicularisation ou vernacularisation langagière, l'emploi alterné des langues ou les pratiques linguistiques dans les communautés plurilingues (y compris les communautés immigrées). Bref, il est question des objets de recherche qui sont importants du point de vue de la société ou d'un groupe social donné et qui permettent de proposer certaines modalités de leur fonctionnement. Ainsi,

la notion de politique linguistique, appliquée en général à l'action d'un État, désigne les choix, les objectifs, les orientations qui sont ceux de cet État en matière de langue(s), choix, objectifs et orientations, suscités en général (mais pas obligatoirement) par une situation intra- ou intercommunautaire préoccupante en matière linguistique (...) ou parfois même ouvertement conflictuelle [...] (Boyer 2010 : 67).

Il s'ensuit alors que la politique linguistique englobe toutes les décisions prises par les autorités concernant l'utilisation des langues sur un territoire donné. 
Cela nous pousse à nous pencher sur deux notions : celle de corpus et celle de statut de la langue, car les actions d'aménagement linguistique oscillent entre ces deux vocables. Par statut, il faut entendre toutes les formes systémiques (dues à la standardisation) de la langue en question tandis que l'usage se réfère à l'emploi social de la langue (Eloy 1997 : 10-12 ; Beacco 2016 : 13-14). Néanmoins, depuis un certain temps, dans le contexte des politiques linguistiques, l'accent est le plus souvent mis sur le côté social de la langue qui couvre, entre autres, les pratiques langagières des communautés (par exemple, les modalités de sélection des variétés langagières), leurs croyances et idéologies ou encore différentes façons d'influencer les pratiques langagières d'une communauté (Beacco 2016 : 20-21).

$\mathrm{Vu}$ le sujet du présent article, il convient de constater que la notion de politique linguistique se réfère également aux systèmes éducatifs, au sens où la politique définit la place occupée par les langues à l'école, de même que leurs modalités d'enseignement/apprentissage. Les auteurs du document du Conseil de l'Europe Guide pour l'élaboration des politiques linguistiques éducatives en Europe (2007) partagent cette opinion en ajoutant l'éducation à un des champs de la politique linguistique. Cette dernière est définie, dans ledit document, comme « une action volontaire, officielle ou militante, destinée à intervenir sur les langues quelles qu'elles soient (nationales, régionales minoritaires, étrangères...) dans leurs formes (les systèmes d'écriture, par exemple), dans leurs fonctions sociales (choix d'une langue comme langue officielle) ou dans leur place d'enseignement. » (Beacco \& Byram 2007 : 17).

Il s'ensuit du passage qui précède que la politique linguistique devrait également couvrir l'enseignement/apprentissage des langues (nationales, régionales, minoritaires et étrangères dans le sens large du terme) tout en tenant compte de leurs caractéristiques résultant à la fois du corpus et du statut. Ce point de vue est repris par le Conseil de l'Europe dont la mission principale est de veiller sur la défense et la promotion des valeurs fondamentales de la communauté dont le multilinguisme fait partie.

\section{LA POLITIQUE LINGUISTIQUE DE L'UNION EUROPEENNE}

Étant donné le caractère hétérogène de la Communauté et la grande mobilité de ses citoyens, le multilinguisme est inscrit de manière permanente dans le quotidien des Européens. Pour cette raison, c'est le caractère multilingue de l'Union européenne qui garantit son fonctionnement cohérent et qui - par voie de conséquence - devrait être protégé et cultivé. Il va donc de soi que c'est le multilinguisme qui devrait se placer au centre de la politique linguistique actuelle, notamment celle qui concerne les systèmes éducatifs. Les auteurs du Cadre 
européen commun de référence (2001) (désormais le CECR) le définissent en tant que « connaissance d'un certain nombre de langues ou la coexistence de langues différentes dans une société. On peut arriver au multilinguisme simplement en diversifiant l'offre de langues dans une école ou un système éducatif donnés, ou en encourageant les élèves à étudier plus d'une langue étrangère, ou en réduisant la place dominante de l'anglais dans la communication internationale. » (CECR 2001 : 11). Il s'ensuit donc que c'est l'école qui est l'endroit privilégié pour la promotion du multilinguisme. En effet, toutes les actions entreprises par les autorités nationales reflètent les axes principaux de la politique linguistique de l'Union européenne, ce qui est surtout visible dans les domaines suivants : enseignement/ apprentissage des langues étrangères vivantes, enseignement/apprentissage des langues régionales et minoritaires, certification des compétences langagières et enseignement d'une matière intégré à une langue (désormais EMILE).

\subsection{Des langues étrangères vivantes}

En réfléchissant sur la politique linguistique promouvant le multilinguisme, il semble nécessaire de souligner l'importance des conclusions de la présidence du Conseil européen, formulées lors du sommet à Barcelone en mars 2002. En effet, les pays membres sont encouragés à modifier leurs politiques éducatives pour « améliorer la maîtrise des compétences de base, notamment par l'enseignement $\mathrm{d}$ 'au moins deux langues étrangères dès le plus jeune âge $»^{1}$. Formulée de cette façon, la conclusion en question change significativement la réalité éducative. D'un côté, l'accent est mis sur le processus d'enseignement/apprentissage précoce des langues étrangères vivantes ; de l'autre, on redéfinit la notion de compétence de communication qui englobe, outre la langue maternelle des apprenants, la connaissance de deux autres langues (Kucharczyk 2018 : 24). Dans les tableaux qui suivent, nous présentons des données statistiques relatives au nombre d'élèves apprenant des langues étrangères lors de leur parcours scolaire obligatoire.

Tableau 1. Pourcentage d'élèves apprenant des LVE à l'école primaire en Europe

\begin{tabular}{|c|c|c|c|c|}
\hline \multirow{2}{*}{$\begin{array}{c}\text { Nombre de langues } \\
\text { apprises au niveau } \\
\text { primaire - UE }\end{array}$} & année 2005 & année 2007 & année 2010 & année 2014 \\
\cline { 2 - 5 } & 32,60 & 29,70 & 21,80 & 16,3 \\
\hline $\mathbf{0}$ & 64,70 & 62,70 & 72,10 & 79,2 \\
\hline $\mathbf{1}$ & 2,80 & 3,10 & 6,10 & 4,6 \\
\hline $\mathbf{2}$ & &
\end{tabular}

Sources : Eurydice (2012 : 58) ; Eurydice (2017 : 165).

${ }^{1}$ https://www.consilium.europa.eu/media/20935/71026.pdf [accès : 27.10.2019]. 
Tableau 2. Pourcentage d'élèves apprenant des LVE à l'école secondaire du 1er cycle en Europe

\begin{tabular}{|c|c|c|c|c|}
\hline \multirow{2}{*}{$\begin{array}{c}\text { Nombre de langues ap- } \\
\text { prises au niveau secon- } \\
\text { daire (1 cycle) - UE }\end{array}$} & année 2005 & année 2007 & année 2010 & année 2014 \\
\cline { 2 - 5 } & 1,2 & 1,2 & 1,8 & 1,4 \\
\hline $\mathbf{0}$ & 52,0 & 44,8 & 37,4 & 38,9 \\
\hline $\mathbf{1}$ & 46,7 & 54,0 & 60,8 & 59,7 \\
\hline
\end{tabular}

Sources : Eurydice (2012: 67) ; Eurydice (2017 : 166).

Tableau 3. Pourcentage d'élèves apprenant des LVE à l'école secondaire du 2e cycle en Europe

\begin{tabular}{|c|c|c|c|c|}
\hline \multirow{2}{*}{$\begin{array}{c}\text { Nombre de langues ap- } \\
\text { prises au niveau secon- } \\
\text { daire (2 cycle) - UE }\end{array}$} & \multicolumn{4}{|c|}{ Pourcentage d'élèves [\%] } \\
\cline { 2 - 5 } & année 2005 & année 2007 & année 2010 & année 2014 \\
\hline $\mathbf{0}$ & 4,6 & 2,5 & 3,6 & 2,9 \\
\hline $\mathbf{1}$ & 33,3 & 34,8 & 36,9 & 28,4 \\
\hline $\mathbf{2}$ & 62,2 & 62,6 & 59,4 & 68,7 \\
\hline
\end{tabular}

Sources : Eurydice (2012: 71); Eurydice (2017: 166).

Les données présentées ci-dessous montrent deux tendances :

- de plus en en plus d'élèves apprennent au moins une langue étrangère à l'école,

- les élèves étudiant au moins deux langues étrangères au niveau secondaire sont de plus en plus nombreux.

On peut donc supposer que la voie au multilinguisme dans le contexte éducatif se voit ouverte, mais comme le remarquent les auteurs du rapport Eurydice, le nombre d'heures de cours de LVE à l'école primaire reste relativement pauvre, ce qui résulte - dans la majorité des cas - des décisions politiques prises par les autorités de l'État.

\subsection{Des langues régionales et minoritaires}

La politique linguistique actuelle accorde également une place importante aux langues régionales et minoritaires qui sont traitées comme une valeur ajoutée à la diversité culturelle et linguistique de l’Union européenne et qui - par voie de conséquence - devraient être protégées vu le risque de leur disparition. Ce point de vue est adopté par la Charte européenne des langues régionales ou minoritaires (1992) qui oblige les pays membres qui l'ont ratifiée à protéger et valoriser les langues régionales et minoritaires présentes sur leur territoire, car ces langues 
font partie intégrante du patrimoine national de la communauté en question. Il va donc de soi que les langues régionales et minoritaires retrouvent leur place légitime dans les systèmes éducatifs, car les pays qui ont ratifié la Charte européenne des langues régionales ou minoritaires sont censés proposer la formation (totale ou partielle) dans une langue minoritaire ou régionale. Il ne s'agit pas seulement des cours de matières non-linguistiques, mais aussi des cours de langues régionales ou minoritaires. Il faut quand même souligner que cette formation ne doit pas se faire au détriment des langues nationales (Kucharczyk 2018 : 39). Les statistiques fournies par le rapport Eurydice (2012) semblent confirmer que les langues minoritaires et régionales gagnent de l'importance dans les systèmes éducatifs européens car, dans presque les deux tiers des pays membres, elles sont intégrées dans les programmes scolaires :

Dans presque les deux tiers des pays, les autorités éducatives centrales recommandent ou exigent que certaines langues régionales ou minoritaires, ou certaines langues dépourvues de territoires, soient incluses dans les programmes des établissements scolaires. Dans beaucoup de pays, toutes les langues bénéficiant d'un statut officiel figurent dans les recommandations ou dans les exigences relatives aux programmes d'études (Eurydice 2012 : 49).

\subsection{La certification des compétences langagières}

La promotion du multilinguisme dans le domaine éducatif est également visible au niveau de la standardisation du processus d'enseignement/apprentissage des langues dans les systèmes éducatifs européens. Il est surtout question du recours aux descripteurs de compétences langagières définis par les auteurs du CECR, qui constituent une sorte de cadre de référence pour la formation des enseignants de LVE, l'élaboration des curricula et des supports didactiques, de même que pour la construction des épreuves certificatives en LVE. Il est nécessaire de souligner que la majorité des pays membres teste le niveau des compétences langagières des apprenants à la fin d'une étape éducative donnée en se référant aux descripteurs fournis par le CECR. Il est alors possible non seulement de comparer les résultats obtenus par les élèves venant de différents pays, mais aussi de comparer les matrices des tests, ce qui peut déboucher sur un échange intéressant concernant la mise en place de la politique du multilinguisme. L'image ci-dessous montre les pays qui recourent aux descripteurs du CECR lors de la certification des compétences langagières des apprenants : 


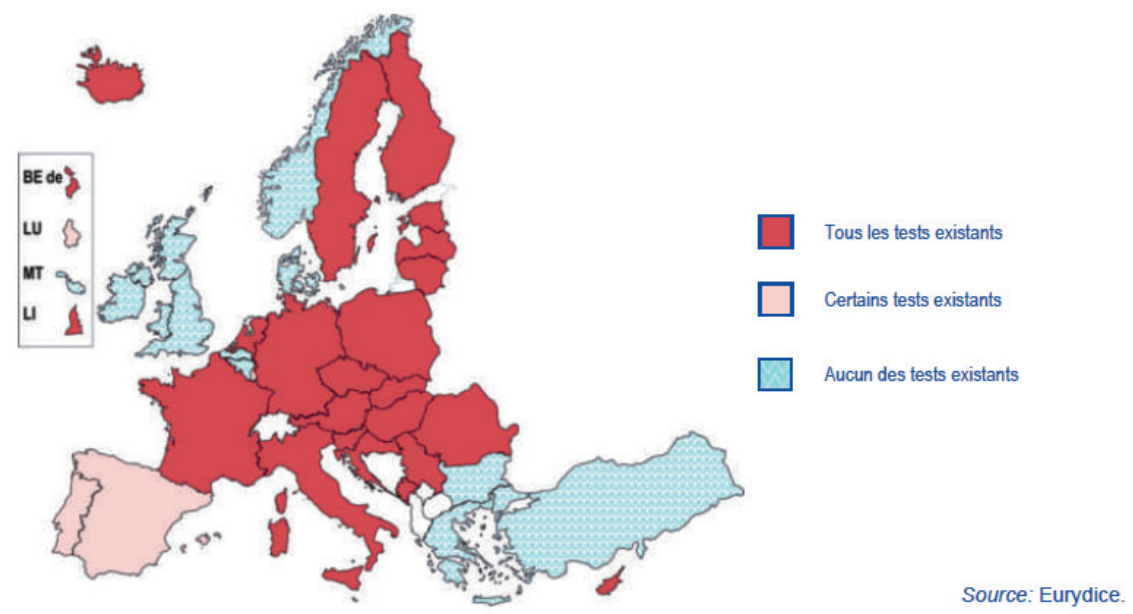

Figure 1. Pays dont les tests de compétences langagières se réfèrent aux descripteurs du CECR Sources : Eurydice (2015: 32).

\subsection{L'enseignement d'une matière intégré à une langue (EMILE)}

Proposer l'enseignement d'une discipline non linguistique dans une langue étrangère, régionale ou minoritaire n'est pas un phénomène nouveau. En effet, encore avant les années 1970, ce type d'enseignement était présent soit dans les régions à situation linguistique particulière comme les régions frontalières ou bilingues, soit dans les grandes villes pour permettre aux apprenants « d'acquérir des compétences linguistiques proches de celles dont disposent les locuteurs natifs » (Eurydice $2006: 7$ ). Cet enseignement, dit bilingue, était un enseignement élitiste, réservé à un groupe très restreint d'apprenants. Depuis les années 1990, on observe des changements dans les pratiques de l'éducation bilingue : «l'accent est mis sur le fait que la langue et la matière non linguistique sont toutes deux objets d'enseignement, sans qu'il y ait de préséance de l'une par rapport à l'autre. » (Eurydice $2006: 7$ ). Cette nouvelle modalité de travail, appelée Enseignement d'une Matière Intégré à une Langue Étrangère, demande une démarche réflexive non seulement de la part des enseignants, mais aussi des autorités qui décident de la politique linguistique du pays.

Les données montrent (Kucharczyk 2018 : 41-42) que, dans une grande majorité des pays européens, l'EMILE fait partie intégrante des systèmes éducatifs soit en tant qu'une des possibilités de parcours scolaire, soit en tant que projet pilote, ce que montre l'image ci-dessous : 


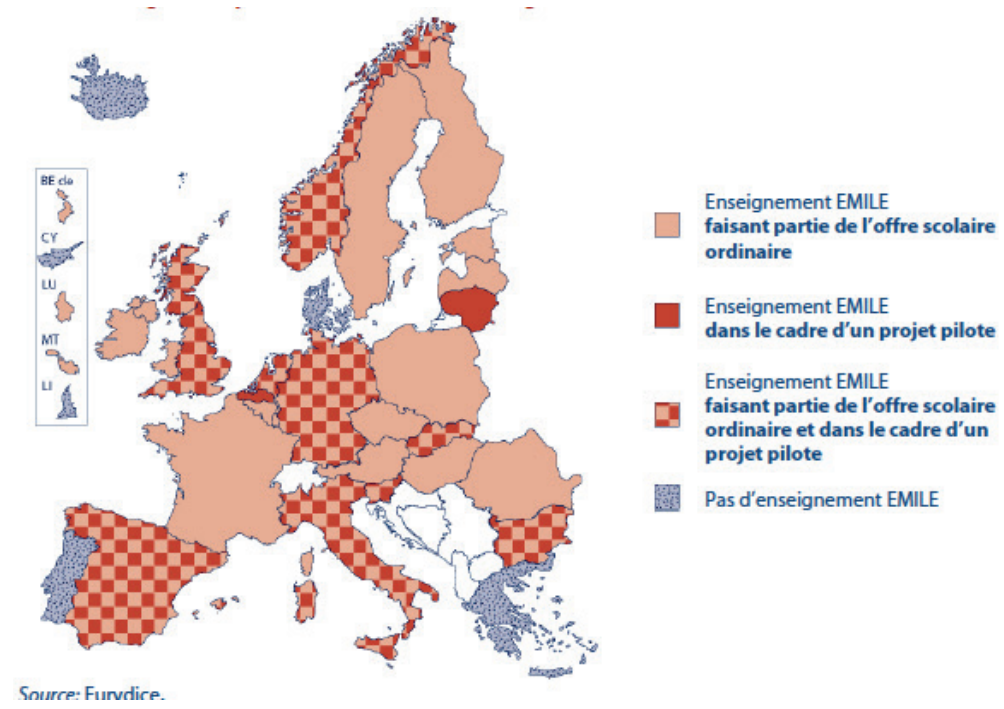

Figure 2. Statut de l'EMILE dans les pays européens Source : EMILE (2006 : 13).

Il est aussi important de souligner que, d'habitude, cette forme d'enseignement se déroule à travers les langues minoritaires ou régionales, ce qui est visible sur l'image ci-dessous :

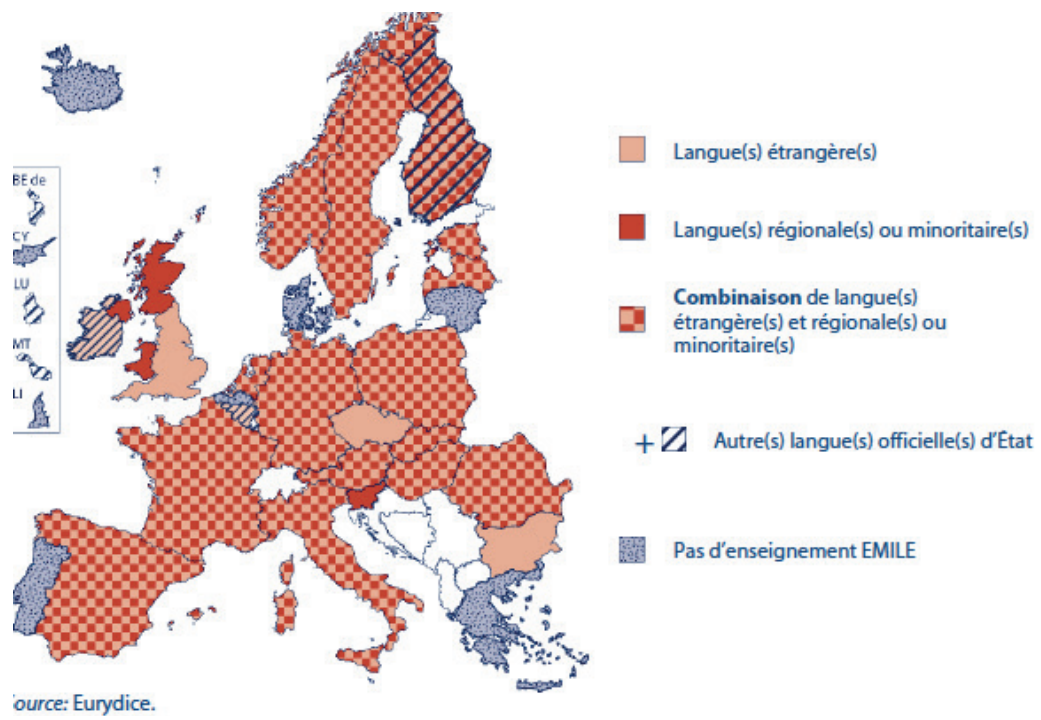

Figure 3. Langues utilisées dans l’EMILE

Source : Eurydice (2006: 17). 


\section{LA POLITIQUE LINGUISTIQUE DE LA POLOGNE}

La Pologne, en tant que membre de la Communauté européenne depuis le mois de mai 2004, suit les axes principaux de la politique européenne, y compris les directives concernant la politique du multilinguisme ; ceci est avant tout visible dans la sphère éducative. Adopter des démarches visant le multilinguisme ne semble pas facile vu la basse variété linguistique et culturelle en Pologne. En effet, la Pologne peut être qualifiée de pays à faible variété linguistique et culturelle pour les raisons suivantes (Wojciechowska 2010 : 67 ; Kucharczyk 2016 : 250) :

- en Pologne, il existe une seule langue officielle, à savoir le polonais (en d'autres termes, c'est la langue qui est utilisée dans la vie publique, de même que dans l'éducation),

- $95 \%$ des personnes habitant sur le territoire polonais se déclarent Polonais,

- 98 \% des habitants de la Pologne considèrent que le polonais est leur langue maternelle et $99 \%$ d'eux utilisent le polonais dans leur quotidien,

- il existe une seule langue régionale en Pologne : le kachoube,

- le nombre d'immigrants vivant sur le territoire de la République de la Pologne est relativement bas.

\subsection{Les langues vivantes étrangères à l'école polonaise}

Il résulte de ce qui précède que c'est l'école, en Pologne, qui est le lieu privilégié pour la promotion du multilinguisme. En effet, les élèves polonophones, lors de leur parcours scolaire, peuvent apprendre jusqu'à trois langues vivantes étrangères (désormais LVE). Ci-dessous, nous montrons l'organisation du processus d'enseignement/apprentissage des LVE à l'école polonaise :

Tableau 4. Organisation du processus d'enseignement/apprentissage des LVE à l'école polonaise

\begin{tabular}{|c|c|c|}
\hline \multicolumn{2}{|r|}{ école primaire } & \multirow{2}{*}{ lycée } \\
\hline classes 1-6 & classes 7-8 & \\
\hline \multicolumn{3}{|l|}{ LVE1 } \\
\hline & LVE2 & \\
\hline \multicolumn{3}{|c|}{ ou bien } \\
\hline \multicolumn{2}{|l|}{ LVE1 } & LVE3 \\
\hline & LVE2 & \\
\hline
\end{tabular}


Depuis l'année scolaire 2009/2010, les élèves polonophones apprennent une langue étrangère depuis leur entrée à l'école primaire à l'âge de 7 ans. Puis, 6 ans plus tard, ils commencent à apprendre une autre LVE dont ils peuvent continuer l'apprentissage à l'école secondaire. Suite à l'implémentation des directives européennes (conclusions du Conseil européen de 2002), le nombre d'élèves apprenant des LVE a significativement augmenté au cours des dernières années, ce que montrent les données dans les tableaux ci-dessous :

Tableau 5. Pourcentage d'élèves apprenant des LVE à l'école primaire en Pologne

\begin{tabular}{|c|c|c|c|c|}
\hline \multirow{2}{*}{$\begin{array}{c}\text { Nombre de langues } \\
\text { apprises au niveau } \\
\text { primaire - PL }\end{array}$} & \multicolumn{4}{|c|}{ Pourcentage d'élèves [\%] } \\
\cline { 2 - 5 } & année 2005 & année 2007 & année 2010 & année 2014 \\
\hline $\mathbf{0}$ & - & 45,5 & 2,3 & 2,3 \\
\hline $\mathbf{1}$ & - & 52,3 & 83,8 & 86,8 \\
\hline $\mathbf{2}$ & - & 1,3 & 13,9 & 11 \\
\hline
\end{tabular}

Sources : Eurydice (2012: 58); Eurydice (2017: 165).

Tableau 6. Pourcentage d'élèves apprenant des LVE à l'école secondaire du 1er cycle en Pologne

\begin{tabular}{|c|c|c|c|c|}
\hline \multirow{2}{*}{$\begin{array}{c}\text { Nombre de langues ap- } \\
\text { prises au niveau secon- } \\
\text { daire (1 cycle) - UE }\end{array}$} & année 2005 & année 2007 & année 2010 & année 2014 \\
\cline { 2 - 5 } & - & 1,9 & 1,8 & 1,7 \\
\hline $\mathbf{0}$ & - & 89,6 & 32,9 & 4,7 \\
\hline $\mathbf{1}$ & - & 8,5 & 65,3 & 93,7 \\
\hline
\end{tabular}

Sources : Eurydice (2012: 67) ; Eurydice (2017: 166).

Tableau 7. Pourcentage d'élèves apprenant des LEV à l'école secondaire du 2e cycle en Pologne

\begin{tabular}{|c|c|c|c|c|}
\hline \multirow{2}{*}{$\begin{array}{c}\text { Nombre de langues ap- } \\
\text { prises au niveau secon- } \\
\text { daire (2 cycle) - UE }\end{array}$} & année 2005 & année 2007 & année 2010 & année 2014 \\
\cline { 2 - 5 } & - & 0,8 & 2,7 & 2,9 \\
\hline $\mathbf{0}$ & - & 19,9 & 24,9 & 28,4 \\
\hline $\mathbf{1}$ & - & 79,3 & 72,4 & 68,7 \\
\hline $\mathbf{2}$ & &
\end{tabular}

Sources : Eurydice (2012: 71); Eurydice (2017: 166).

Néanmoins, force est de constater que, même si les apprenants polonophones apprenant deux ou plus de LVE sont de plus en plus nombreux, c'est l'anglais qui reste la LVE la plus souvent choisie par eux, ce que montrent les données présentées ci-dessous : 
Tableau 8. Popularité des LVE à l'école polonaise

\begin{tabular}{|l|c|c|c|c|c|}
\hline \multirow{3}{*}{ Langue } & \multicolumn{5}{|c|}{$\begin{array}{c}\text { Année scolaire } \\
\text { pourcentage d'élèves [\%] }\end{array}$} \\
\cline { 2 - 6 } & $\mathbf{2 0 0 5 / 2 0 0 6}$ & $\mathbf{2 0 1 0 / 2 0 1 1}$ & $\mathbf{2 0 1 2 / 2 0 1 3}$ & $\mathbf{2 0 1 5 / 2 0 1 6}$ & $\mathbf{2 0 1 9 / 2 0 2 0}$ \\
\hline Anglais & $\mathbf{6 5 , 9}$ & 89,0 & 93,2 & 94,9 & 91,8 \\
\hline Français & 3,3 & 2,9 & 3,0 & 2,4 & 2,8 \\
\hline Allemand & 33,6 & 36,4 & 38,2 & 33,7 & 35,4 \\
\hline russe & 6,1 & 4,8 & 4,9 & 3,9 & 4,4 \\
\hline
\end{tabular}

Source : Oświata i wychowanie w roku szkolnym 2015/2016 (2016 : 102) ; Oświata i wychowanie w roku szkolnym 2019/2020 $(2020: 21)$.

\subsection{La certification en langues vivantes étrangères à l'école polonaise}

La promotion de la politique du multilinguisme à l'école polonaise est également visible dans l'organisation des examens externes en LVE. Comme le curriculum polonais pour les LVE se réfère aux niveaux de référence proposés par les concepteurs du CECR, il va de soi que les épreuves nationales le font aussi. L'organisation des examens externes en LVE en Pologne est montrée dans le tableau qui suit:

Tableau 9. Épreuves en LVE à l’école polonaise

\begin{tabular}{|c|c|c|c|c|}
\hline École & Épreuve & $\begin{array}{l}\text { Niveau du } \\
\text { CECR }\end{array}$ & $\begin{array}{c}\text { Épreuve } \\
\text { obligatoire }\end{array}$ & Remarques \\
\hline primaire & $\begin{array}{l}\text { la même épreuve } \\
\text { pour tous les appre- } \\
\text { nants }\end{array}$ & $\mathrm{A} 2+/ \mathrm{B} 1$ & oui & - \\
\hline \multirow{3}{*}{ secondaire } & $\begin{array}{l}\text { baccalauréat - } \\
\text { niveau de base }\end{array}$ & $\mathrm{B} 1+/ \mathrm{B} 2$ & oui & $\begin{array}{l}\text { Cette épreuve est obligatoire pour } \\
\text { les élèves qui ont décidé de se } \\
\text { présenter au baccalauréat. }\end{array}$ \\
\hline & $\begin{array}{l}\text { baccalauréat - } \\
\text { niveau avancé }\end{array}$ & $\mathrm{B} 2+/ \mathrm{C} 1$ & non & - \\
\hline & $\begin{array}{l}\text { baccalauréat - } \\
\text { niveau bilingue }\end{array}$ & $\mathrm{C} 1+/ \mathrm{C} 2$ & non & - \\
\hline
\end{tabular}

\subsection{Les langues minoritaires et régionales à l'école polonaise}

Nous venons de constater que la Pologne se caractérise par un bas niveau de diversité linguistique et culturelle. Néanmoins, sur le territoire de la République de Pologne, on distingue des minorités nationales et ethniques (Kucharczyk 2020 : 92). Parmi les minorités nationales, on distingue les minorités suivantes 
(dans l'ordre alphabétique) : arménienne, allemande, biélorusse, juive, lithuanienne, russe, slovaque, tchèque et ukrainienne. En ce qui concerne les minorités ethniques, elles sont moins nombreuses : Karaïmes (pl. Karaimi), Lemkos (pl. Łemkowie), Roms (pl. Romowie) et Tatars (pl. Tatarzy).

Selon la loi polonaise, la minorité - qu' elle soit nationale ou ethnique - est moins nombreuse que l'ensemble des citoyens polonais et se distingue par sa propre langue, culture et tradition qu'elle cultive et protège. De plus, pour que la minorité donnée puisse jouir du statut officiel de minorité, elle doit être présente sur le territoire polonais depuis au moins 100 ans. La seule différence entre la minorité nationale et la minorité ethnique consiste en l'identification à la nation organisée au sein de son propre territoire : si les minorités nationales s'y identifient, ce n'est pas le cas pour les minorités ethniques.

Dans le tableau qui suit, nous présentons les données relatives aux minorités nationales et ethniques présentes sur le territoire polonais :

Tableau 10. Minorités nationales et ethniques en Pologne

\begin{tabular}{|c|c|c|c|c|c|}
\hline \multicolumn{2}{|c|}{ Minorité } & $\begin{array}{c}\text { Appartenance } \\
\text { à la minorité } \\
\text { nationale/eth- } \\
\text { nique déclarée } \\
\text { en } 2002\end{array}$ & $\begin{array}{c}\text { Utilisation } \\
\text { de la langue } \\
\text { minoritaire au } \\
\text { foyer déclarée } \\
\text { en } 2002 \\
\end{array}$ & $\begin{array}{c}\text { Appartenance } \\
\text { à la minorité } \\
\text { nationale/eth- } \\
\text { nique déclarée } \\
\text { en } 2012 \\
\end{array}$ & $\begin{array}{c}\text { Utilisation } \\
\text { de la langue } \\
\text { minoritaire au } \\
\text { foyer déclarée } \\
\text { en } 2012\end{array}$ \\
\hline \multirow{9}{*}{$\begin{array}{l}\text { minorités } \\
\text { nationales }\end{array}$} & allemande & 147094 & 196841 & 144236 & 96461 \\
\hline & arménienne & 262 & 321 & 1683 & 1847 \\
\hline & biélorusse & 47640 & 43878 & 40226 & 26448 \\
\hline & juive & 1055 & 243 & 7353 & 411 \\
\hline & lituanienne & 5639 & 5696 & 5696 & 5303 \\
\hline & russe & 3244 & 12125 & 8796 & 19805 \\
\hline & slovaque & 1710 & 794 & 2739 & 765 \\
\hline & tchèque & 386 & 1226 & 2831 & 1451 \\
\hline & ukrainienne & 27172 & 21055 & 38795 & 24539 \\
\hline \multirow{4}{*}{$\begin{array}{l}\text { minorités } \\
\text { ethniques }\end{array}$} & Karaïmes & 43 & - & 314 & - \\
\hline & Lemkos & 5850 & 5605 & 9640 & 6279 \\
\hline & Roms & 12731 & 15657 & 16723 & 14468 \\
\hline & Tatars & 447 & 9 & 1828 & 9 \\
\hline
\end{tabular}

Source : Kucharczyk (2020 : 93-94).

De plus, sur le territoire de la République, il existe une seule langue régionale: le kachoube (pl. kaszubski). Par langue régionale, on entend une langue qui est utilisée sur le territoire d'un pays par des citoyens qui sont en minorité et qui n'est 
pas un dialecte ou une langue des migrants ${ }^{2}$. La minorité kachoube est relativement nombreuse, ce que montrent les données présentées dans le tableau ci-dessous :

Tableau 11. Minorité kachoube en Pologne : 2002 et 2012

\begin{tabular}{|c|c|c|c|c|}
\hline $\begin{array}{c}\text { Minorité } \\
\text { régionale }\end{array}$ & $\begin{array}{c}\text { Appartenance à la } \\
\text { minorité région- } \\
\text { ale déclarée en } \\
\mathbf{2 0 0 2}\end{array}$ & $\begin{array}{c}\text { Utilisation de } \\
\text { la langue mi- } \\
\text { noritaire au foyer } \\
\text { déclarée en 2002 }\end{array}$ & $\begin{array}{c}\text { Appartenance à la } \\
\text { minorité région- } \\
\text { ale déclarée en } \\
\mathbf{2 0 1 2}\end{array}$ & $\begin{array}{c}\text { Utilisation de } \\
\text { la langue mi- } \\
\text { noritaire au foyer } \\
\text { déclarée en 2012 }\end{array}$ \\
\hline Kachoubes & 5062 & 52665 & 214600 & 108100 \\
\hline
\end{tabular}

Source : GUS (2002 et 2011).

Selon la loi polonaise, l'État est censé maintenir chez les apprenants leur identité « minoritaire », ce qui revient à leur donner la possibilité d'apprendre la langue minoritaire. En effet, trois curricula ont été conçus (Kucharczyk 2020 : 95-96) qui balisent le processus d'enseignement/apprentissage des langues minoritaires en définissant ses objectifs et - par voie de conséquence - les objectifs des examens externes en langues minoritaires. Il est question des curricula suivants :

- le curriculum pour les langues de minorité nationale (pl. podstawa programowa języka mniejszości narodowej),

- le curriculum pour les langues de minorité ethnique (pl. podstawa programowa języka mniejszości etnicznej),

- le curriculum pour la langue kachoube (pl. podstawa programowa języka kaszubskiego).

Dans le tableau qui suit, nous présentons des données concernant le nombre d'élèves étudiant les langues minoritaires lors de leur parcours scolaire en Pologne:

Tableau 12. Élèves apprenant des langues minoritaires en Pologne

\begin{tabular}{|l|c|c|c|c|}
\hline & \multicolumn{4}{|c|}{ Nombre total d'élèves } \\
\hline \multicolumn{1}{|c|}{ langue } & année 2008/2009 & année 2009/2010 & année 2010/2011 & année 2016/2017 \\
\hline allemand & 23616 & 23481 & 25076 & 44 191 \\
\hline arménien & 16 & 15 & 35 & pas de données \\
\hline biélorusse & 3438 & 3037 & 3041 & 2710 \\
\hline hébreu & 315 & 310 & 327 & pas de données \\
\hline kachoube & 9776 & 10658 & 13632 & 19473 \\
\hline
\end{tabular}

${ }^{2}$ D'après Ustawa o mniejszościach narodowych i etnicznych oraz języku regionalnym $z$ dn. 6 stycznia 2005. http:/ / prawo.sejm.gov.pl/isap.nsf/download.xsp/WDU20050170141/U/D20050141Lj.pdf [accès : 19.05.2018] 
Radosław Kucharczyk

\begin{tabular}{|l|c|c|c|c|}
\hline & \multicolumn{4}{|c|}{ Nombre total d'élèves } \\
\hline \multicolumn{1}{|c|}{ langue } & année 2008/2009 & année 2009/2010 & année 2010/2011 & année 2016/2017 \\
\hline karaïm & pas de données & pas de données & pas de données & pas de données \\
\hline lemkovien & 288 & 288 & 268 & pas de données \\
\hline lituanien & 681 & 660 & 675 & pas de données \\
\hline romani & pas de données & pas de données & pas de données & 2224 \\
\hline russe & pas de données & pas de données & pas de données & pas de données \\
\hline slovaque & 217 & 197 & 196 & pas de données \\
\hline tatar & pas de données & pas de données & pas de données & pas de données \\
\hline tchèque & pas de données & pas de données & pas de données & pas de données \\
\hline ukrainien & pas de données & pas de données & pas de données & 1807 \\
\hline
\end{tabular}

Source : GUS (2016).

\subsection{L'EMILE à l'école polonaise}

La promotion du multilinguisme à l'école polonaise est également possible grâce à l'introduction de l'EMILE dans le système éducatif polonais. En effet, tout établissement scolaire qui propose l'enseignement d'au moins deux matières dans deux langues - le polonais et une LVE - est appelé un établissement bilingue qui réalise les principes de l'EMILE (Pawlak 2015 : 5). Dans une LVE peuvent être enseignées les disciplines suivantes : biologie, chimie, physique, géographie (sauf les cours consacrés aux sujets relatifs à la géographie de la Pologne), histoire universelle (ceci dit, sans l'histoire de la Pologne), mathématiques et autres matières dites complémentaires.

L'enseignement de type EMILE a avant tout lieu au lycée, mais - suite à la dernière réforme éducative de l'année 2016/2017 - il peut être aussi réalisé dans les deux dernières classes de l'école primaire. Dans la majorité des cas, l'EMILE se réalise dans une LVE. Dans le tableau qui suit, nous présentons les données relatives au nombre de lycées bilingues et d'élèves qui y suivent les cours d'EMILE :

Tableau 13. Établissements bilingues en Pologne en 2015

\begin{tabular}{|l|c|c|c|}
\hline \multicolumn{1}{|c|}{ Voïvodie } & $\begin{array}{c}\text { Nombre } \\
\text { d'établissements }\end{array}$ & $\begin{array}{c}\text { Nombre de classes } \\
\text { «bilingues » }\end{array}$ & Nombre d'élèves \\
\hline Mazovie & 25 & 97 & 2660 \\
\hline Silésie & 13 & 56 & 1621 \\
\hline Poméranie & 10 & 38 & 1077 \\
\hline Grande-Pologne & 8 & 33 & 894 \\
\hline voïvodie d'Opole & 7 & 11 & 235 \\
\hline
\end{tabular}




\begin{tabular}{|l|c|c|c|}
\hline \multicolumn{1}{|c|}{ Voïvodie } & $\begin{array}{c}\text { Nombre } \\
\text { d'établissements }\end{array}$ & $\begin{array}{c}\text { Nombre de classes } \\
\text { «bilingues » }\end{array}$ & Nombre d'élèves \\
\hline Basse-Silésie & 6 & 21 & 503 \\
\hline voïvodie de Łódź & 6 & 19 & 478 \\
\hline Poméranie Occidentale & 5 & 19 & 448 \\
\hline Coü̈avie-Poméranie & 4 & 15 & 435 \\
\hline Petite-Pologne & 3 & 18 & 499 \\
\hline voïvodie de Lublin & 2 & 9 & 252 \\
\hline Podlachie & 2 & 5 & 119 \\
\hline Basses-Carpates & 1 & 1 & 13 \\
\hline voïvodie de Lubusz & 1 & 2 & 126 \\
\hline Varmie-Mazurie & 1 & 4 & 0 \\
\hline Sainte-Croix & 0 & 0 & $\mathbf{9 4 0 3}$ \\
\hline TOTAL & $\mathbf{9 4}$ & $\mathbf{3 4 8}$ & \\
\hline
\end{tabular}

Source : Pawlak (2015: 14).

Pour ce qui est des langues d'enseignement dans les établissements bilingues, la vedette revient sans nul doute à l'anglais ; viennent ensuite : l'allemand, l'espagnol, le français, l'italien et le russe, ce que montre le tableau ci-dessous :

Tableau 14. Les langues dans les établissements bilingues en Pologne en 2015

\begin{tabular}{|l|c|c|c|}
\hline $\begin{array}{c}\text { Langue } \\
\text { d'enseignement }\end{array}$ & $\begin{array}{c}\text { Nombre } \\
\text { d'établissements }\end{array}$ & Nombre de classes & Nombre d'élèves \\
\hline anglais & 56 & 190 & 5144 \\
\hline allemand & 22 & 51 & 1307 \\
\hline espagnol & 15 & 54 & 1511 \\
\hline français & 12 & 44 & 1278 \\
\hline italien & 2 & 2 & 48 \\
\hline russe & 2 & 2 & 48 \\
\hline
\end{tabular}

Source : Pawlak (2015: 16).

\section{BILAN}

Nous venons de constater que la notion de multilinguisme est un des axes principaux de la politique de l'Union européenne, notamment dans le contexte éducatif. Il va donc de soi que c'est non seulement l'enseignement/apprentissage des LVE qui doit être valorisé dans le parcours scolaire obligatoire, mais que les langues minoritaires devraient y retrouver leur place légitime. La République de Pologne, membre de la Communauté européenne depuis 2004, réalise, elle 
aussi, les directives concernant la politique du multilinguisme. Vu le faible degré de diversité linguistique et culturelle de la Pologne, la mise en place de ces recommandations se fait avant tout à l'école. Les élèves polonophones ont la possibilité d'apprendre jusqu'à trois LVE lors de leur parcours scolaire. Ils peuvent également suivre l'enseignement de type EMILE dans une langue choisie (anglais, allemand, espagnol, français, italien ou russe). De plus, le curricula pour les LVE et - par voie de conséquence - les examens externes de LVE sont reliés au CECR, ce qui garantit la transparence des acquis linguistiques des apprenants. Il faut également souligner que les langues minoritaires (y compris le kachoube - la langue régionale présente sur le territoire polonais) retrouvent leur place légitime dans le système éducatif polonais aussi bien au niveau du processus d'enseignement/apprentissage que de celui de la certification des compétences langagières. Bref, il résulte de ce qui précède que la mise en place de la politique du multilinguisme dans un pays où la diversité linguistique et culturelle est relativement basse est possible à condition que les autorités de l'État prévoient, dans le cadre des systèmes éducatifs, des modalités de fonctionnement appropriées.

\section{BIBLIOGRAPHIE}

Beacco, J.-C. (2016). École et politiques linguistiques. Pour une gestion de la diversité linguistique. Paris: Didier.

Beacco, J.-C. / Byram, M. (2007). Guide pour l'élaboration des politiques linguistiques éducatives en Europe. Version intégrale. Strasbourg: Conseil de l'Europe.

Boyer, H. (2010). Les politiques linguistiques. Mots. Les langages du politique, 94, 67-74.

Cadre européen de référence pour les langues: apprendre, enseigner, évaluer (2001). Strasbourg: Conseil de l'Europe.

Chiffres clés de l'enseignement des langues à l'école en Europe (2012). Bruxelles: Eurydice, unité européenne.

Chiffres clés de l'enseignement des langues à l'école en Europe (2017). Luxembourg: Office des publications de l'Union européenne.

Conclusion de la présidence du Conseil européen du mars 2002. https:/ / www.consilium.europa.eu/ media/20935/71026.pdf [accès: 27.11.2019].

Eloy, J.-M. (1997). «Aménagement» ou «politique» linguistique? Mots. Les langages du politique, 57, $7-22$.

Kucharczyk, R. (2016). Kontekst nauczania języków obcych w Polsce - glottodydaktyczne implikacje dla drugich języków obcych. Linguodidactica, 20, 195-212.

Kucharczyk, R. (2018). Nauczanie języków obcych a dydaktyka wielojęzyczności (na przykładzie języka francuskiego jako drugiego jezzyka obcego). Lublin: Werset.

Kucharczyk, R. (2020). La place des langues dites majoritaires/minoritaires dans l'enseignement primaire et secondaire en Pologne. Journal of Applied Linguistics, 33, 90-103. 
La Charte européenne des langues régionales ou minoritaires (1992). https://rm.coe.int/168007c07e [accès: 5.06.2020].

L'école en Europe - Édition 2012. Rapport Eurydice (2012). Bruxelles: Agence exécutive Éducation, Audiovisuel et Culture.

L'école en Europe - Édition 2017 (2017) Rapport Eurydice. Luxembourg: Office des publications de l'Union européenne.

L'enseignement d'une matière intégré à une langue étrangère (EMILE) à l'école en Europe. Rapport Eurydice (2006). Bruxelles: Eurydice, unité européenne.

Les langues dans l'enseignement secondaire: un aperçu des tests nationaux en Europe - 2014/2015. Rapport Eurydice (2015). Luxembourg: Office des Office des publications de l'Union européenne.

Oświata i wychowanie 2015/2016 (2016). Warszawa: Główny Urząd Statystyczny.

Oświata i wychowanie 2019/2020 (2020). Warszawa: Główny Urząd Statystyczny.

Pawlak, M. (2015). Edukacja dwujęzyczna w polskich szkołach. Raport ewaluacyjny. Warszawa: Ośrodek Rozwoju Edukacji.

Struktura narodowo-etniczna, językowa i wyznaniowa ludności Polski. Narodowy Spis Powszechny Ludności i Mieszkań 2011 (2015). Warszawa: Główny Urząd Statystyczny.

Wojciechowska, B. (2010). Wielojęzyczność w polskim kontekście edukacyjnym. Neofilolog, 35, 63-74.

Wyniki Narodowego Spisu Powszechnego Ludności i Mieszkań 2002 w zakresie deklarowanej narodowości oraz jezzyka używanego w domu. https://stat.gov.pl/spisy-powszechne/narodowe-spisypowszechne/narodowy-spis-powszechny-2002/wyniki-narodowego-spisu-powszechnego2002-narodowosci-oraz-jezyka/ [accès: 19.05.2018].

Received: 06.12.2020; revised: 15.10 .2021

\author{
RADOSŁAW KUCHARCZYK \\ Uniwersytet Warszawski \\ r.kucharczyk@uw.edu.pl \\ ORCID: 0000-0002-1861-1358
}

